\title{
MULTIPLE HOLOMORPHS OF FINITELY GENERATED ABELIAN GROUPS
}

\author{
BY
}

W. H. MILLS

The object of this paper is to determine all cases in which two or more finitely generated abelian groups have the same holomorph(1). Let $G$ and $G^{\prime}$ be finitely generated abelian groups and let $H$ be the holomorph of $G$. Then it will be shown that $H$ is the holomorph of $G^{\prime}$ if and only if $G^{\prime}$ is an invariant maximal-abelian subgroup of $H$ isomorphic to $G$. All such subgroups of $H$ are determined. There are at most four. If $G$ does not contain any elements of order 2, or if $G$ has at least three independent generators of infinite order, then $G$ itself is the only such subgroup $\left({ }^{2}\right)$.

1. Definitions. Let $G$ be a group. If $\sigma$ and $\tau$ are two automorphisms of $G$, then $\sigma \tau$ is defined to be the automorphism such that $(\sigma \tau) g=\sigma(\tau g)$ for all $g \in G$. Under this composition the automorphisms of $G$ form a group $A$. Consider the set $H$ of all pairs $(g, \sigma), g \in G, \sigma \in A$. We define a composition in $H$ by

$$
(a, \sigma)(b, \tau)=(a \sigma b, \sigma \tau) \text {. }
$$

Under this composition the set $H$ forms a group. If $e$ is the identity of $G$ and $I$ is the identity of $A$, then $(e, I)$ is the identity of $H$. Furthermore the inverse of $(a, \sigma)$ is $\left(\sigma^{-1} a^{-1}, \sigma^{-1}\right)$. The group $H$ is called the holomorph ${ }^{(3)}$ of $G$. The mapping $g \rightarrow(g, I)$ gives an imbedding of $G$ in the group $H$. We identify the element $g$ in $G$ with the element $(g, I)$ in $H$. Then $G$ is an invariant subgroup of $H$. If $G$ is abelian, then it is a maximal-abelian subgroup of $H$, that is, an abelian subgroup not properly contained in any abelian subgroup of $H$.

Received by the editors February 2, 1951.

(1) This problem was proposed by J. G. Wendel for the case of finite abelian groups. Wendel considered the group algebra of a finite abelian group $G$ over the field of complex numbers. He then considered the group $J$ of all norm preserving automorphisms of this group algebra, where the norm is the sum of the absolute values of the coefficients. Wendel then observed that the group $J$ was the holomorph of $G$, and asked whether the group $G$ was determined by the abstract group $J$. The methods of $\$ 2$ are due, in part, to Wendel who first obtained the fundamental identity (6).

(2) This generalizes the results of G. A. Miller [1]. (Numbers in brackets refer to the references cited at the end of the paper.) Miller used the classical definition of the holomorph as the set of all permutations that transform a regular permutation group into itself. He determined all cases in which two or more isomorphic finite abelian groups have the same holomorph. A slight discrepancy between Miller's paper and this paper is due to the fact that different definitions of the holomorph are used.

$\left(^{3}\right)$ Various other definitions of the holomorph have been given. For example the holomorph of a group $G$ is the group of transformations of $G$ generated by the automorphisms of $G$ and the left (or right) multiplications, $g \rightarrow a g(g \rightarrow g a)$. 
More generally, if $\eta$ is any isomorphism of $H$ onto a group $H^{\prime}$, and if $G^{\prime}$ is the image of $G$ under this isomorphism, then $H^{\prime}$ is said to be the holomorph of $G^{\prime}$.

2. Several identities. We shall now establish several identities for later use. Let $H$ be the holomorph of an abelian group $G$, and let $S$ be an invariant Abelian subgroup of $H$. Let $(a, \sigma)$ be a fixed element of $S, b$ an arbitrary element of $G$, and $\tau$ an arbitrary element of $A$. Since $S$ is invariant,

$$
(e, \tau)(a, \sigma)(e, \tau)^{-1}=\left(\tau a, \tau \sigma \tau^{-1}\right) \in S .
$$

Therefore, since $S$ is commutative,

$$
(a, \sigma)\left(\tau a, \tau \sigma \tau^{-1}\right)=\left(\tau a, \tau \sigma \tau^{-1}\right)(a, \sigma) .
$$

Comparing first components we obtain

$$
a \sigma \tau a=\tau a \tau \sigma \tau^{-1} a .
$$

Therefore

$$
\text { if } \tau a=a \text {, then } \sigma a=\tau \sigma a .
$$

Let $\theta$ be the automorphism of $G$ that sends each element into its inverse. Put $\lambda=\sigma^{-1} \theta$. Then, since $\sigma$ and $\theta$ both commute with $\sigma$, it follows that $\lambda \sigma \lambda^{-1}=\sigma$. Substituting $\tau=\lambda$ in (1) gives $e=\sigma^{-1} a^{-1} \sigma a$. Applying $\sigma$ we obtain

$$
\sigma^{2} a=a .
$$

Now $S$ contains $(a, \sigma)(e, \lambda)(a, \sigma)(e, \lambda)^{-1}=\left(e, \sigma^{2}\right), b^{-1}(a, \sigma) b(a, \sigma)^{-1}=\sigma b / b$, and hence $(\sigma a / a)^{-1}(a, \sigma)^{2}\left(e, \sigma^{2}\right)^{-1}=a^{2}$. We have established:

$$
\left(e, \sigma^{2}\right) \in S, \quad \sigma b / b \in S, \quad \text { and } a^{2} \in S .
$$

Furthermore $S$ contains $(e, \tau)(\sigma b / b)(e, \tau)^{-1}=\tau(\sigma b / b)$. Now, since $S$ is abelian, $(a, \sigma)$ commutes with $\tau(\sigma b / b)$. It follows that

$$
\sigma \tau(\sigma b / b)=\tau(\sigma b / b) \text {. }
$$

If we put $\tau=I$ in (5), then we obtain

$$
\sigma(\sigma b / b)=\sigma b / b .
$$

From (6) it follows, by induction on $n$, that

$$
\sigma^{n} b=b(\sigma b / b)^{n}
$$

and

$$
(a, \sigma)^{n}=\left(a^{n}(\sigma a / a)^{n(n-1) / 2}, \sigma^{n}\right),
$$

for all positive integral $n$. Finally, comparing (3) and (7) we have

$$
(\sigma a / a)^{2}=e .
$$


3. Additional notation. We shall now suppose that $G$ and $G^{\prime}$ are finitely generated abelian groups with holomorphs $H$ and $H^{\prime}$ respectively. Suppose that $G$ is isomorphic to an invariant subgroup $G$ of $H^{\prime}$, and that $G^{\prime}$ is isomorphic to an invariant subgroup $G^{\prime}$ of $H$. This condition is clearly satisfied if $G$ and $G^{\prime}$ have isomorphic holomorphs.

Now since $G$ and $G^{\prime}$ are finitely generated abelian groups, they can be written as direct products

$$
\begin{aligned}
G & =G(\infty) \times G\left(p_{1}\right) \times \cdots \times G\left(p_{M}\right), \\
G^{\prime} & =G^{\prime}(\infty) \times G^{\prime}\left(p_{1}\right) \times \cdots \times G^{\prime}\left(p_{M}\right),
\end{aligned}
$$

where $G(\infty)$ and $G^{\prime}(\infty)$ are groups with no elements of finite order except for the identity; $p_{1}, \cdots, p_{M}$ are distinct primes; and $G\left(p_{i}\right)$ and $G^{\prime}\left(p_{i}\right)$ consist of all elements, of $G$ and $G^{\prime}$ respectively, whose orders are integral powers of $p_{i}$. By allowing the groups $G\left(p_{i}\right)$ and $G^{\prime}\left(p_{i}\right)$ to contain only the identity, we can assume that the same primes occur in the decompositions (10) and (11), and that 2 is one of these primes. The groups $G\left(p_{i}\right)$ and $G^{\prime}\left(p_{i}\right)$ are finite abelian invariant subgroups of $H$. We now put

$$
\begin{gathered}
F(\infty)=\prod_{i=1}^{M} G\left(p_{i}\right), \quad F^{\prime}(\infty)=\prod_{i=1}^{M} G^{\prime}\left(p_{i}\right), \\
F(2)=G(\infty) \prod_{p_{i} \neq 2} G\left(p_{i}\right) .
\end{gathered}
$$

Since the groups $G^{\prime}\left(p_{i}\right)$ and $G^{\prime}$ are invariant abelian subgroups of $H$, it follows that the identities of $\$ 2$ hold if $(a, \sigma)$ is an element of one of these groups.

4. Elements of maximal prime power order. Let $p$ be one of the primes $p_{1}, \cdots, p_{M}$. Let $p^{m}$ and $p^{m^{\prime}}$ be the maximal orders of the elements of $G(p)$ and $G^{\prime}(p)$ respectively. We need the following:

Lemma 1. If $(a, \sigma) \in G^{\prime}, b \in G(p)$, and $m \geqq 1$, then $(\sigma b / b)^{p^{m-1}}=e$.

Proof. Since $b \in G(p)$, it follows that $\sigma b / b \in G(p)$, and therefore $(\sigma b / b)^{p^{m}}$ $=e$. Suppose $(\sigma b / b)^{p^{m-1}} \neq e$. Then $b^{p^{m-1}} \neq e$. Hence $b$ and $\sigma b / b$ are both elements of $G(p)$ of maximal order. Therefore there exists an automorphism $\tau \in A$ such that $\tau(\sigma b / b)=b$. By (5) we have $\sigma b=b$ or $\sigma b / b=e$, which implies that $(\sigma b / b)^{p^{m-1}}=e$.

Lemma 2. $m=m^{\prime}$.

Proof. Let $(a, \sigma)$ be an element of $G^{\prime}(p)$. Then

$$
(a, \sigma)^{p^{\prime \prime}}=e=(e, I) .
$$

Comparing second components, we see that $\sigma^{p^{n^{\prime}}}=I$. If $b \in G$, then from (7) we have $(\sigma b / b)^{p^{m^{\prime}}}=e$. Hence $\sigma b / b \in G(p)$ and therefore $(\sigma b / b)^{p^{m}}=e$. Since $b$ is an arbitrary element of $G,(7)$ yields $\sigma^{p^{m}}=I$. Now by (8) 


$$
e=(a, \sigma)^{p^{m^{\prime}+1}}=\left(a^{p^{\prime \prime+1}}(\sigma a / a)^{p^{m^{\prime}+1}\left(p^{m^{\prime}+1}-1\right) / 2}, \sigma^{p^{\prime \prime+1}}\right)=a^{p^{\prime \prime+1}} .
$$

Hence $a \in G(p)$. Therefore $a^{p^{m}}=e$. By Lemma 1 we have $(\sigma a / a)^{p^{m-1}}=e$ if $m \geqq 1$. It follows that

$$
(a, \sigma)^{p^{m}}=(\sigma a / a)^{p^{m}\left(p^{m-1}\right) / 2}=e,
$$

for any value of $m$. Now (13) holds for all $(a, \sigma) \in G^{\prime}(p)$. Hence $m^{\prime} \leqq m$. By symmetry $m \leqq m^{\prime}$. Therefore $m^{\prime}=m$.

Let $N(p)$ and $N^{\prime}(p)$ denote the number of elements of $G(p)$ and $G^{\prime}(p)$ respectively of maximal order $p^{m}$.

LEMMA 3. $N^{\prime}(p) \geqq N(p)$.

Proof. If $m=0$, then $G(p)$ and $G^{\prime}(p)$ both consist of the identity alone, and $N^{\prime}(p)=N(p)=1$. We suppose that $m>0$. Let $(a, \sigma)$ be a fixed element of $G^{\prime}(p)$ of order $p^{m}$. In the proof of Lemma 2 we established that $\sigma^{p^{m}}=I$ and $a^{p^{m}}=e$. If the order of $\sigma$ is $p^{m}$, then by (7) there exists a $b \in G$ such that $\sigma b / b$ has order $p^{m}$. By $(4), \sigma b / b \in G^{\prime}(p)$. Hence $G^{\prime}(p)$ contains an element $(a, \sigma)$ of order $p^{m}$, where $\sigma^{p^{m-1}}=I$. We distinguish two cases:

Case I. The order of $a$ is $p^{m}$. Here we can choose $\tau_{1}, \tau_{2}, \cdots, \tau_{N(p)}$ so that $\tau_{i} a$ runs through the $N(p)$ elements of $G(p)$ of order $p^{m}$. Then

$$
\left(e, \tau_{i}\right)(a, \sigma)\left(e, \tau_{i}\right)^{-1}=\left(\tau_{i} a, \tau_{i} \sigma \tau_{i}^{-1}\right)
$$

are $N(p)$ distinct elements of $G^{\prime}(p)$ of order $p^{m}$. Thus in this case $N^{\prime}(p) \geqq N(p)$.

Case II. $a^{p^{m-1}}=e$. In this case we have, by (8),

$$
e \neq(a, \sigma)^{p^{m-1}}=(\sigma a / a)^{p^{m-1}\left(p^{m-1-1) / 2}\right.} \text {. }
$$

Clearly $(\sigma a / a)^{p^{m-1}}=e$, and by (9) we have $(\sigma a / a)^{2}=e$. It follows that $p=2$ and $m=2$. Furthermore the order of $\sigma a / a$ is exactly 2 . Therefore the order of $\sigma$ and the order of $a$ are both 2. If $a$ is a square, say $a=g^{2}$, then the order of $\sigma g / g$ is 4 , which contradicts Lemma 1. Therefore $a$ is not a square. Put $b=\sigma a / a$. If $b$ is not a square, then there exists an automorphism $\tau$ such that $\tau(\sigma a / a)=\tau b=a$, and then (5) yields $\sigma a=a$, which is a contradiction. Therefore $b$ is a square. Put $b=c^{2}$. Then the order of $c$ is 4 and $\sigma a=a b=a c^{2}$. Let $B_{1}$ and $B_{2}$ be the cyclic groups generated by $c$ and $a$ respectively. We can write $G(2)$ as a direct product, $G(2)=B_{1} \times B_{2} \times \cdots \times B_{t}$, where the $B_{i}$ are cyclic groups of order 2 and 4 . We shall show that $t=2$. Suppose $t \geqq 3$, and let $d$ be a generator of $B_{3}$.

(i) Suppose $d$ has order 4. Choose $\tau \in A$ such that $\tau a=a, \tau c=d$. Then by (2) we have $\sigma a=\tau \sigma a$, or $a c^{2}=a d^{2}$, or $c^{2}=d^{2}$, which is a contradiction. Therefore $B_{1}$ is the only one of the groups $B_{i}$ of order 4 .

Now let $g$ and $\tau$ be arbitrary elements of $G$ and $A$ respectively. Then, since $\sigma^{2}=I$, it follows that $(\sigma g / g)^{2}=e$. By (5) we have $\sigma \tau(\sigma g / g)=\tau(\sigma g / g)$ and therefore $\tau(\sigma g / g) \neq a$. Since this is true for arbitrary $\tau$, it follows that $\sigma g / g$ is either $e$ or $c^{2}$. Therefore 


$$
\tau(\sigma g / g)=\sigma g / g
$$

for all $g \in G, \tau \in A$. Hence (1) yields

$$
\sigma \tau a / \tau a=\tau\left(\sigma \tau^{-1} a / \tau^{-1} a\right)=\sigma \tau^{-1} a / \tau^{-1} a .
$$

(ii) Suppose $d$ has order 2. Choose $\tau$ such that $\tau a=d, \tau^{-1} a=a d$. Then from (16) we have $\sigma d / d=\sigma(a d) /(a d)=c^{2} \sigma d / d$, or $c^{2}=e$, a contradiction. Therefore $t=2$ and $G(2)=B_{1} \times B_{2}$. Hence $N(2)=4$.

Now $\sigma(a c) /(a c)=c^{2} \sigma c / c$, and $\sigma c / c$ is either $e$ or $c^{2}$. By replacing, if necessary, $c$ by $a c$ we can assume that $\sigma c=c$. Now choose $\xi \in A$ such that $\xi c=a c$, $\xi a=a$, and $\xi f=f$ if $f \in F(2)$. Put $\rho=\xi \sigma \xi^{-1}$. Then $\rho a=a c^{2}$ and $\rho c=c^{3}$. Clearly $\rho \neq \sigma$, and therefore

$$
\begin{gathered}
(a, \sigma), \quad(a, \sigma)^{3}=\left(a c^{2}, \sigma\right), \\
(e, \xi)(a, \sigma)(e, \xi)^{-1}=(a, \rho), \\
(a, \rho)^{3}=\left(a c^{2}, \rho\right)
\end{gathered}
$$

are four distinct elements of $G^{\prime}(2)$ of order 4 . It follows that $N^{\prime}(2) \geqq 4=N(2)$. This completes the proof of Lemma 3.

By symmetry we have $N(p) \geqq N^{\prime}(p)$. Thus we have:

Lemma 4. $N^{\prime}(p)=N(p)$.

We shall now re-examine the two cases of Lemma 3 in the light of Lemma 4 .

5. The case $p$ odd. If $p \neq 2$, then Case II is impossible and we have only Case I left. Then we have $(a, \sigma) \in G^{\prime}(p)$ where $a$ is of order $p^{m}$. Since $G^{\prime}(p)$ is an invariant abelian subgroup of $H$, we can apply (4), and hence $a^{2} \in G^{\prime}(p)$. Since $a$ is an element of odd order, it follows that $a \in G^{\prime}(p)$. Therefore $(e, \tau) a(e, \tau)^{-1}=\tau a \in G^{\prime}(p)$ for all $\tau \in A$. Now the elements $\tau a$ generate $G(p)$. Hence $G(p) \subseteq G^{\prime}(p)$. By symmetry $G^{\prime}(p) \subseteq G(p)$. Thus we have proved:

Lemma 5. If $p \neq 2$ then $G^{\prime}(p)=G(p)$.

6. The case $p=2$. We write $G(\infty)$ and $G(2)$ as the direct product of fixed nontrivial cyclic groups:

$$
G(\infty)=G_{1} \times G_{2} \times \cdots \times G_{k}, \quad G(2)=C_{1} \times C_{2} \times \cdots \times C_{t}
$$

where $k \geqq 0, t \geqq 0$, and the order of $C_{i}$ is greater than or equal to that of $C_{i+1}$. Let $g_{i}$ be a generator of the group $G_{i}$. Let $\alpha$ and $\beta$ be generators of $C_{1}$ and $C_{2}$ respectively, and let $u, v$, and $w$ be the orders of $C_{1}, C_{2}$, and $C_{3}$ respectively. If $t<3$ it is understood that $w=1$; if $t<2$, that $v=1, \beta=e$; and if $t=0$, that $u=1, \alpha=e$. Then $u, v$, and $w$ are powers of 2 and $u \geqq v \geqq w$. We put

$$
F_{i}=\prod_{i=j+1}^{t} C_{i}, \quad i=1,2,
$$


where, if $t \leqq j, F_{j}$ is understood to consist of the identity alone.

Suppose $p=2$. Then $2^{m}=u$. If $u=1$ then $G^{\prime}(2)=G(2)$ trivially. We suppose that $u>1$. We have either Case I or Case II.

Case I. $G^{\prime}(2)$ contains an element $(a, \sigma)$ of order $u$, where $a$ has order $u$ and $\sigma^{u / 2}=I$. Here the $N(2)$ elements (14) constitute the entire set of elements of $G^{\prime}(2)$ of order $u$. Now every element of $G(2)$ of order $u$ occurs once and only once as the first component of one of the elements (14). Therefore $G^{\prime}(2)$ contains an element of order $u$ with $\alpha$ as its first component, say $(\alpha, \psi)$. Furthermore if $\left(\alpha_{1}, \sigma_{1}\right)$ and $\left(\alpha_{1}, \sigma_{2}\right)$ are elements of $G^{\prime}(2)$ of order $u$ with the same first component, then $\sigma_{1}=\sigma_{2}$ and the order of $\alpha_{1}$ is $u$. In particular if $\tau$ is an automorphism of $G$ such that $\tau \alpha=\alpha$, then $(e, \tau)(\alpha, \psi)(e, \tau)^{-1}=\left(\alpha, \tau \psi \tau^{-1}\right)$ and $(\alpha, \psi)$ are such elements, and hence $\tau \psi \tau^{-1}=\psi$. Thus we have

$$
\text { if } \tau \alpha=\alpha \text {, then } \tau \psi=\psi \tau \text {. }
$$

Suppose $(e, \tau) \in G^{\prime}(2)$. Then it follows that the order of $(e, \tau)$ is less than $u$, and hence the order of $(\alpha, \psi)(e, \tau)=(\alpha, \psi \tau)$ is $u$. Therefore $\psi \tau=\psi$ or $\tau=I$. Combined with Lemma 5 this yields:

Lemma 6. If Case I holds for $p=2$, then $F^{\prime}(\infty)$ contains no elements of the form $(e, \tau), \tau \neq I$.

In particular (4) gives us $\left(e, \psi^{2}\right) \in G^{\prime}(2)$ and hence $\psi^{2}=I$. Then (7) yields $(\psi g / g)^{2}=e$ for all $g \in G$.

Suppose first that $k$, the number of independent generators of infinite order, is positive. Put $h=\psi g_{1} / g_{1}$. Then $\psi g_{1}=g_{1} h, h^{2}=e$, and hence $h \in G(2)$ $\subseteq F(\infty)$. Let $f$ be either an element of $F(\infty)$ or one of the infinite generators $g_{i}, i \geqq 2$. Then we can choose $\tau$ such that $\tau g_{1}=g_{1} f$ and $\tau b=b$ if $b \in F(\infty)$. Then by (18) we have $\tau \psi=\psi \tau$. Now $\psi \tau g_{1}=\psi\left(g_{1} f\right)=g_{1} h \psi f$, and $\tau \psi g_{1}=\tau\left(g_{1} h\right)$ $=g_{1} h f$. Hence $\psi f=f$.

If $k \geqq 2$, then $\psi g_{i}=g_{i}$ if $i \geqq 2$, and by interchanging the roles of $g_{1}$ and $g_{2}$ we have $\psi g_{1}=g_{1}$. Also, since $\psi f=f$ if $f \in F(\infty)$, we have $\psi=I$, and hence $G(2) \subseteq G^{\prime}(2)$. Since $N^{\prime}(2)=N(2)$, it follows that $G(2)=G^{\prime}(2)$.

If $k=1$ then we have $\psi f=f$ for all $f \in F(\infty)$, and we need only determine $\psi g_{1}=g_{1} h$. Now if $\tau \alpha=\alpha$ and $\tau g_{1}=g_{1}$, then

$$
g_{1} \tau h=\tau\left(g_{1} h\right)=\tau \psi g_{1}=\psi \tau g_{1}=\psi g_{1}=g_{1} h .
$$

Thus $\tau h=h$ for all $\tau$ for which $\tau \alpha=\alpha, \tau g_{1}=g_{1}$. Since $h^{2}=e$ this is possible if and only if $h=\alpha^{q}$, where $q=0$ or $q=u / 2$. By (4), $h=\alpha^{q} \in G^{\prime}(2)$. If $q=u / 2$ then $\alpha \notin G^{\prime}(2)$ and hence $u>2$. Thus $q$ is even. If $b \in G(2)$ let $\psi_{b}$ be the automorphism such that

$$
\psi_{b} g_{1}=g_{1} b^{q}, \quad \text { and } \quad \psi_{b} f=f \quad \text { if } f \in F(\infty) .
$$

Now $(\alpha, \psi)$ and its conjugates generate $G^{\prime}(2)$. It follows that $G^{\prime}(2)$ consists of the elements $\left(b, \psi_{b}\right), b \in G(2)$. In this case $G^{\prime}(2)$ is isomorphic to $G(2)$. 
Finally suppose $k=0$. By (18) we see that $\tau \psi \alpha=\psi \alpha$ for all $\tau$ for which $\tau \alpha=\alpha$. This can be true only if $\psi \alpha$ is a power of $\alpha$, say

$$
\psi \alpha=\alpha^{r},
$$$$
1 \leqq r<u .
$$

Now we know that $(\psi \alpha / \alpha)^{2}=e$ or $\alpha^{2(r-1)}=e$. It follows that either $r=1$ or $r=1+u / 2$. If $u=2$, then of necessity $r=1$. If $u=4$ then $e \neq(\alpha, \psi)^{2}=\alpha^{1+r}$. Hence $r=1$ here also. Thus we have:

$$
\text { If } u \leqq 4 \text {, then } r=1 \text {. If } u>4 \text {, then } r=1 \text { or } r=1+u / 2 \text {. }
$$

Now if $\tau \alpha=\alpha$ and $\tau \beta=\beta$, then $\tau \psi \beta=\psi \tau \beta=\psi \beta$. Therefore $\psi \beta=\alpha^{s} \beta^{R}$ for suitable $s$ and $R$, where we can suppose $0 \leqq s<u$. If $f=\alpha^{u / v}$ or if $f \in F_{2}$ we can choose $\tau$ such that $\tau \alpha=\alpha, \tau \beta=\beta f$. Then by (18), $\psi \tau=\tau \psi$. Now $\psi \tau \beta=\psi(\beta f)=\alpha^{s} \beta^{R} \psi f$, and $\tau \psi \beta=\tau\left(\alpha^{8} \beta^{R}\right)=\alpha^{8} \beta^{R} f^{R}$. Thus

$$
\psi f=f^{R} .
$$

Now $f=\alpha^{u / v}$ gives $\alpha^{r u / v}=\alpha^{R u / v}$, whence $r \equiv R(\bmod v)$. Thus we have $\beta^{r}=\beta^{R}$ and without loss of generality we can suppose that $R=r$. Now $(\psi \beta / \beta)^{2}=e$ and hence $\alpha^{2 s 2} \beta^{(r-1)}=e$, which yields $\alpha^{2 s}=e$. Hence we have

$$
\psi \beta=\alpha^{s} \beta^{r}, \text { where } s=0 \text { or } s=u / 2 .
$$

Also from (22) if $f \in F_{2}$, then $\psi f=f^{r}$. Now if $v=w$ we can interchange the roles of $C_{2}$ and $C_{3}$ to obtain $\psi \beta=\beta^{r}$, or $s=0$.

$$
\text { If } v=w \text {, then } s=0 .
$$

Now choose $\tau$ such that $\tau \alpha=\alpha \beta, \tau \beta=\beta$. Then $\tau^{-1} \alpha=\alpha / \beta$ and (1) gives $\alpha \psi(\alpha \beta)$ $=\alpha \beta \tau \psi(\alpha / \beta)$ or

$$
\alpha^{1+r+8} \beta^{r}=\alpha^{1+r-8} \beta^{1-8} .
$$

Since $\alpha^{2 s}=e$ we have $\beta^{r}=\beta^{1-s}$ or $\beta^{r-1}=\beta^{-s}=\beta^{s}$. Therefore

$$
\text { if } u=v \text {, then } s=r-1 \text {. }
$$

Since $u \geqq v \geqq w$, it follows from (24) and (25) that if $u=w$, then $s=0$ and $r=1$. If $u>w$ and if $f \in F_{2}$, then $\psi f=f^{r}=f$. Hence in any case

$$
\text { if } f \in F_{2} \text {, then } \psi f=f^{r}=f \text {. }
$$

By (10) and (12), $G=G(2) \times F(2)$ and every element of $F(2)$ has odd order. Hence

$$
\text { if } f \in F(2) \text {, then } \psi f=f \text {. }
$$

We note that $r$ and $s$ determine $\psi$ completely. We have four possibilities:

(i) $r=1, s=0$. This is possible for all values of $u, v$, and $w$.

(ii) $r=1, s=u / 2$. By (24) and (25) this is possible only if $u>v>w$.

(iii) $r=1+u / 2, s=0$. By (21) and (25) we have $u>v, u>4$. 
(iv) $r=1+u / 2, s=u / 2$. By (21) and (24) we have $u>4, v>w$. In other words:

(A) If $u=v=w ; u=v \leqq 4$; or if $u \leqq 4$ and $v=w$; then $r=1, s=0$.

(B) If $u=4, v=2, w=1 ; r=1$, and $s=0$ or $s=u / 2$.

(C) If $u>v=w$ and $u>4$; then $s=0$, and $r=1$ or $r=1+u / 2$.

(D) If $u=v>w$ and $u>4$; then $r=1$ and $s=0$, or $r=1+u / 2$ and $s=u / 2$.

(E) If $u>v>w$ and $u>4$; then $r=1$ or $r=1+u / 2$, and $s=0$ or $s=u / 2$.

Now if $f \in F_{1}$ we can choose $\phi \in A$ such that $\phi \alpha=\alpha f$, and $\phi g=g$ if $g \in F_{1}$ or if $g \in F(2)$. Then $G^{\prime}(2)$ contains $(e, \phi)(\alpha, \psi)(e, \phi)^{-1}$. Therefore $G^{\prime}(2)$ also contains

$$
(e, \psi)(\alpha, \psi)^{-1}(e, \phi)(\alpha, \psi)(e, \phi)^{-1}(e, \psi)^{-1}=\left(f, \phi \psi \phi^{-1} \psi^{-1}\right) .
$$

If $f \in F_{2}$ then $\phi \psi=\psi \phi$ and hence $f \in G^{\prime}(2)$. Therefore $F_{2} \subseteq G^{\prime}(2)$. Now put $f=\beta$ and $\omega=\phi \psi \phi^{-1} \psi^{-1}$. Then $(\beta, \omega) \in G^{\prime}(2)$ and

$$
\begin{aligned}
& \omega \alpha=\alpha^{1+s} \beta^{s}, \quad \omega \beta=\beta^{1+8}, \\
& \omega g=g \text { if } g \in F_{2} \text { or if } g \in F(2) .
\end{aligned}
$$

On the other hand, let $G(2, r, s)$ be the group generated by $(\alpha, \psi),(\beta, \omega)$, and the elements of $F_{2}$, where $\psi$ and $\omega$ are defined by (20), (23), (26), (27), and (28). Then if $k=0, G^{\prime}(2)$ is one of the groups $G(2, r, s)$, where the possible values of $r$ and $s$ are given by (A), (B), (C), (D), and (E). We note that this holds whether or not $u=1$. If $u=4, v=2$, and $w=1$, then Case II leads to one additional possibility.

Case II. $a^{u / 2}=e, \sigma^{u / 2}=I$. Here we have $u=2^{m}=4, v=2$, and $w=1$. Furthermore $N^{\prime}(2)=N(2)=4$. The four elements (17) are the only elements of $G^{\prime}(2)$ of order 4.

Suppose $k \geqq 1$ and put $h=\sigma g_{1} / g_{1}$. Then $h^{2}=e$ and (15) gives us $\tau h=h$ for all $\tau \in A$. Furthermore

$$
\rho g_{1}=\xi \sigma \xi^{-1} g_{1}=\xi \sigma g_{1}=\xi\left(g_{1} h\right)=g_{1} h=\sigma g_{1} .
$$

Choose $\tau$ so that $\tau f=f$ if $f \in F(\infty)$ and $\tau g_{1}=g_{1} a$. Then $(e, \tau)^{-1}(a, \sigma)(e, \tau)$ $=\left(a, \tau^{-1} \sigma \tau\right)$ is an element of $G^{\prime}(2)$ of order 4 . Hence $\tau^{-1} \sigma \tau$ is either $\sigma$ or $\rho$. Therefore

$$
g_{1} h=\tau^{-1} \sigma \tau g_{1}=\tau^{-1} \sigma\left(g_{1} a\right)=\tau^{-1}\left(g_{1} h a c^{2}\right)=g_{1} h c^{2},
$$

which is a contradiction. Therefore $k=0$, and $G$ is a finite group in this case.

The four elements (17) generate a group of order 8 isomorphic to $G(2)$. This group must be $G^{\prime}(2)$. Moreover $(a, \sigma)$ and $(e, \sigma \rho)$ are a set of independent generators of this group. Furthermore $\alpha^{2}=c^{2}$ and $\beta$ must be either $a$ or $a c^{2}$. Therefore $G^{\prime}(2)$ contains the elements $(\beta, \sigma)$ and $(\beta, \rho)$. Also $\sigma \beta=\rho \beta=\beta c^{2}$ $=\beta \alpha^{2}$. Let $\psi$ be the automorphism such that

$$
\psi \alpha=\alpha, \quad \psi \beta=\beta \alpha^{2}, \text { and } \quad \psi f=f \text { if } f \in F(2) .
$$


Then $\psi=\sigma$ or $\psi=\rho$. Hence $(\beta, \psi) \in G^{\prime}(2)$. Now if we put $\omega=\sigma \rho$, then

$$
\omega g=g^{3} \quad \text { if } \quad g \in G(2), \quad \omega f=f \text { if } f \in F(2) .
$$

Then we see that $(\beta, \psi)$ and $(e, \omega)$ are a set of independent generators of $G^{\prime}(2)$.

On the other hand if $u=4, v=2$, and $w=1$, let $\psi$ and $\omega$ be defined by (29) and (30). Then let $\bar{G}(2)$ be the group generated by $(\alpha, \psi)$ and $(e, \omega)$. We see that if Case II holds, then $p=2, k=0$, and $G^{\prime}(2)=\bar{G}(2)$. Thus in all cases we have:

LemMa 7. $G^{\prime}(2)$ is isomorphic to $G(2)$.

7. The groups $G(\infty)$ and $G^{\prime}(\infty)$. We shall now study the group $G^{\prime}(\infty)$. We let $k$ and $k^{\prime}$ be the number of independent generators of $G(\infty)$ and $G^{\prime}(\infty)$ respectively. If $k=0$, then $G$ is a finite group, $H$ is a finite group, and hence $G^{\prime}$ is a finite group. Thus when $k=0$, it follows that $k^{\prime}=0$, and $G^{\prime}(\infty)=G(\infty)$ since both groups consist of the identity alone. We now suppose that $k>0$. Then clearly $k^{\prime}>0$. Let $(a, \sigma)$ be an element of $G^{\prime}$ of infinite order. Then either $a$ or $\sigma$ has infinite order. If $a$ has infinite order then, by (4), $a^{2}$ is an element of $G^{\prime}$ of infinite order. If $\sigma$ has infinite order, then there exists an element $b \in G$ such that $\sigma b / b$ has infinite order. By (4), $\sigma b / b \in G^{\prime}$. In either case $G \cap G^{\prime}$ contains an element $g$ of infinite order. Let $Q$ be the common maximal order of the elements of $F(\infty)$ and $F^{\prime}(\infty)$. Then $g^{Q} \in G(\infty) \cap G^{\prime}(\infty)$. Now $g^{Q}$ and its conjugates form a subgroup of $G(\infty)$ with $k$ independent generators. This subgroup is also a subgroup of $G^{\prime}(\infty)$. Hence $k^{\prime} \geqq k$. By symmetry $k \geqq k^{\prime}$, and hence $k^{\prime}=k$. Therefore $G^{\prime}(\infty)$ is isomorphic to $G(\infty)$. This result, combined with Lemmas 5 and 7, proves the following:

THEOREM 1. If $G$ and $G^{\prime}$ are finitely generated abelian groups, each isomorphic to an invariant subgroup of the holomorph of the other, then $G$ is isomorphic to $G^{\prime}$.

COROLLARY. If $G$ and $G^{\prime}$ are finitely generated abelian groups with isomorphic holomorphs, then $G$ and $G^{\prime}$ are isomorphic.

8. Multiple holomorphs. Let $H$ and $H^{*}$ be the holomorphs of the groups $G$ and $G^{*}$ respectively. Suppose that $H$ and $H^{*}$ are isomorphic and that $\eta^{\prime}$ is an isomorphism of $H^{*}$ onto $H$. Put $G^{\prime}=\eta^{\prime} G^{*}$, the image of $G^{*}$ under the isomorphism $\eta^{\prime}$. We can regard $H$ as the holomorph of $G^{\prime}$. Thus $H$ is simultaneously the holomorph of $G$ and $G^{\prime}$. We call $H$ a multiple holomorph ${ }^{4}$ ) of the groups $G$ and $G^{\prime}$.

Now suppose that $H$ is a multiple holomorph of the finitely generated

(4) This definition differs from the definition of G. A. Miller [1]. Miller defined the multiple holomorph of $G$ as the group obtained from $H$ by adjoining certain elements that transform $G$ into its conjugates. 
abelian groups $G$ and $G^{\prime}$. Then by the corollary to Theorem 1, $G$ and $G^{\prime}$ are isomorphic. Hence there is an automorphism $\eta$ of $H$ that sends $G$ onto $G^{\prime}$. Furthermore $G^{\prime}$ is an invariant maximal-abelian subgroup of $H$ isomorphic to $G$. We shall now determine all such subgroups of $H$.

9. Invariant maximal-abelian subgroups. Let $G$ be any finitely generated abelian group with holomorph $H$, and $G$ be any invariant abelian subgroup of $H$ isomorphic to $G$. Then by putting $G^{\prime}=G^{\prime}=G$ we can apply the results and notations of the preceding sections.

Suppose first that $k=0$. Then $G$ is the product of the groups $G^{\prime}(p)$. When $p \neq 2$, then $G^{\prime}(p)=G(p)$. Hence $G=G^{\prime}(2) F(2)$. We put $G(r, s)=G(2, r, s) F(2)$ and $\bar{G}=\bar{G}(2) F(2)$. Then we see that $G$ is either $\bar{G}$ or one of the groups $G(r, s)$. We note that $\bar{G}$ is defined only if $u=4, v=2$, and $w=1$. The possible values of $r$ and $s$ are given by (A) through (E). It can be easily shown that the groups $\bar{G}$ and $G(r, s)$ are invariant maximal-abelian subgroups of $H$, under the above mentioned restrictions.

Now we suppose that $k>0$. Suppose that $G$ contains an element of the form $(e, \tau)$. As before we let $Q$ be the common maximal order of the elements of $F(\infty)$ and $F^{\prime}(\infty)$. Then if $\tau$ has infinite order, $(e, \tau)^{Q}$ adjoined to $G(\infty)$ $\cap G^{\prime}(\infty)$ gives a subgroup of $G^{\prime}(\infty)$ with $k+1$ independent generators. Hence $\tau$ has finite order, and $(e, \tau) \in F^{\prime}(\infty)$. Now since $k>0$, Case I holds for $p=2$, and by Lemma 6 we have $\tau=I$. We have proved:

Lemma 8. If $k>0$, then $G$ contains no elements of the form $(e, \tau), \tau \neq I$.

Let $(a, \sigma)$ be any element of $G$. By $(4),\left(e, \sigma^{2}\right) \in G$, and Lemma 8 gives us $\sigma^{2}=I$. Let $(a, \tau)$ be another element of $G$ with the same first component. Then $G$ contains $(a, \sigma)^{-1}(a, \tau)=\left(e, \sigma^{-1} \tau\right)$. Therefore $\sigma=\tau$ by Lemma 8 . Thus we have proved:

Lemma 9. If $k>0,(a, \sigma) \in G$, and $(a, \tau) \in G$, then $\sigma=\tau$ and $\sigma^{2}=I$.

It follows at once that if $(a, \sigma) \in G$ and $g \in G$, then $\sigma g^{2}=g^{2}$. Hence $(a, \sigma)$ commutes with $g^{2}$. We now impose the further condition on $G$ that it be a maximal-abelian subgroup of $H$. Then we see that $g^{2} \in G$ for all $g \in G$. Let $K$ be the group of all elements of the form $g^{2} f, g \in G, f \in F^{\prime}(\infty)$. Then $K$ is a subgroup of $G$ and an invariant subgroup of $H$.

Lemma 10. If $k>0$, then $K$ is a proper subgroup of $G$.

Proof. $G$ is a maximal-abelian subgroup of $H$. Therefore it is sufficient to find an element $(g, \sigma)$ that does not belong to $K$ but that commutes with all elements of $K$. Since $K$ does not contain any element with first coefficient $g_{1}$, it is sufficient to find an element $\left(g_{1}, \sigma\right)$ that commutes with all elements of $K$. Now if $k \geqq 2$, then $G^{\prime}(p)=G(p)$ for all $p$. Hence $F^{\prime}(\infty)=F(\infty), K \subseteq G$, and $g_{1}$ is an element with the desired properties. If $k=1$, then $G^{\prime}(p)=G(p)$ for all $p \neq 2$, and $G^{\prime}(2)$ consists of the elements $\left(b, \psi_{b}\right), b \in G(2), \psi_{b}$ defined by (19). 
In this case let $\nu$ be the automorphism such that $\nu g=g^{1+q}$ if $g \in G(2)$, and $\nu f=f$ if $f \in F(2)$. Then $\left(g_{1}, \nu\right)$ is an element that commutes with all elements of $K$. This completes the proof of Lemma 10.

Let $(g, \sigma)$ be an element of $H$ that is not an element of $K$. We may suppose that $g=\prod g_{i}^{n_{i}}$, where each $n_{i}$ is either 0 or 1 . At least one of the $n_{i}$ must be 1 . Hence there exists a $\tau \in A$ such that $\tau g=g_{1}$. Hence $(e, \tau)(g, \sigma)(e, \tau)^{-1}$ $=\left(g_{1}, \tau \sigma \tau^{-1}\right)$ is an element of $G$, but not of $K$. Without loss of generality let $g=g_{1}$. Now if $\tau g_{1}=g_{1}$, then $(e, \tau)\left(g_{1}, \sigma\right)(e, \tau)^{-1}=\left(g_{1}, \tau \sigma \tau^{-1}\right)$ and $\left(g_{1}, \sigma\right)$ are both elements of $G$. Hence, by Lemma 9 , we see that

$$
\text { if } \tau g_{1}=g_{1} \text {, then } \tau \sigma=\sigma \tau \text {. }
$$

Suppose $k \geqq 2$. Let $f \in F(\infty)$ or $f=g_{j}, j \neq 2$. Then there exists a $\tau \in A$ such that $\tau g_{1}=g_{1}, \tau g_{2}=f g_{2}$, and $\tau b=b$ if $b \in F(\infty)$. Then by (31) we have $\tau \sigma=\sigma \tau$. Now

$$
\begin{gathered}
\tau \sigma g_{2}=\tau\left(g_{2}\left(\sigma g_{2} / g_{2}\right)\right)=f g_{2}\left(\sigma g_{2} / g_{2}\right)=f \sigma g_{2}, \\
\sigma \tau g_{2}=\sigma\left(f g_{2}\right)=\sigma f \sigma g_{2} .
\end{gathered}
$$

Hence $\sigma f=f$. In particular we see that $k \geqq 2$ implies that $\sigma f=f$ for all $f \in F(\infty)$.

If $k \geqq 3$, then $\sigma g_{j}=g_{j}$ for all $j \neq 2$. By interchanging the roles of $g_{2}$ and $g_{3}$ we can show that $\sigma g_{2}=g_{2}$. Thus if $k \geqq 3$, then $\sigma=I$ and $G=G$.

Let $Z$ be the center of $H$. If $u=v$, then $Z$ consists of $e$ alone. If $u>v$, then $Z$ consists of $e$ and $\alpha^{u / 2}$. We note that $Z \subseteq G(2)$ and that $Z$ contains at most two elements. $Z$ consists of all elements of $G(2)$ that are left invariant by all automorphisms of $G(2)$.

Now suppose that $k=2$. Then $F^{\prime}(\infty)=F(\infty)$. Furthermore $\sigma f=f$ for all $f \in F(\infty)$ and $\sigma g_{1}=g_{1}$. Put $\sigma g_{2}=g_{2} h$. Then $h^{2}=e$. Now if $\tau g_{1}=g_{1}$ and $\tau g_{2}=g_{2}$, then by (31), $\sigma \tau=\tau \sigma$ and

$$
g_{2} h=\sigma g_{2}=\sigma \tau g_{2}=\tau \sigma g_{2}=\tau\left(g_{2} h\right)=g_{2} \tau h,
$$

or $h=\tau h$. Therefore $h$ is left invariant by all automorphisms of $G(2)$. It follows that $h \in Z$.

Now let $z$ be an arbitrary element of $Z$ and define $\sigma_{1}$ and $\sigma_{2}$ as follows:

$$
\begin{gathered}
\sigma_{i} f=f \quad \text { if } \quad f \in F(\infty), \quad \sigma_{i} g_{i}=g_{i}, \\
\sigma_{i} g_{j}=g_{j} z
\end{gathered}
$$$$
\text { if } i \neq j \text {. }
$$

Let $G(z)$ be the group generated by $\left(g_{1}, \sigma_{1}\right),\left(g_{2}, \sigma_{2}\right)$ and the elements of $F(\infty)$. Then $G(z)$ is an invariant maximal-abelian subgroup of $H$ isomorphic to $G$.

We see that if $k=2$, then $G=G(z)$ for suitable $z \in Z$.

Suppose $k=1$. Then $G^{\prime}(2)$ consists of the elements $\left(b, \psi_{b}\right), b \in G\left({ }^{2}\right), \psi_{b}$ defined by (19). Now $\left(g_{1}, \sigma\right)\left(b, \psi_{b}\right)=\left(b, \psi_{b}\right)\left(g_{1}, \sigma\right)$ since $G^{\prime}$ is commutative. Comparing the first components we get

$$
g_{1} \sigma b=b \psi_{b} g_{1}=g_{1} b^{1+q},
$$


or $\sigma b=b^{1+a}$ for all $b \in G(2)$. If $c \in G(p)$, where $p \neq 2$, then $\sigma c=c$. Hence we need only determine $\sigma g_{1}$. Put $\sigma g_{1}=g_{1} h$. Then $h^{2}=e$. Now by (2) if $\tau g_{1}=g_{1}$, then $\sigma g_{1}=\tau \sigma g_{1}$ which implies that $g_{1} h=\tau\left(g_{1} h\right)$ or $h=\tau h$. Hence $h \in Z$.

On the other hand let $z \in Z$, and if $u \leqq 2$ let $q=0$, if $u>2$ let $q=0$ or $q=u / 2$. Let $\psi_{b}$ be defined by (19) and $\sigma$ by

$$
\begin{gathered}
\sigma g_{1}=g_{1} z, \quad \sigma b=b^{1+q} \text { if } \quad b \in G(2), \\
\sigma c=c \quad \text { if } \quad c \in G(p),
\end{gathered}
$$$$
p \neq 2 \text {. }
$$

Let $G(q, z)$ be the group generated by $\left(g_{1}, \sigma\right) ;\left(b, \psi_{b}\right), b \in G(2)$; and the elements of the groups $G(p), p \neq 2$. Then $G(q, z)$ is an invariant maximal-abelian subgroup of $H$.

If $k=1$, then $G$ is of the form $G(q, z)$.

Thus we have determined the invariant maximal-abelian subgroups of $H$ isomorphic to $G$. We see that there are at most four such subgroups. If $G$ does not contain any elements of order 2, or if $G$ contains at least three independent generators of infinite order, then $G$ is the only such subgroup.

10. Groups with the same holomorph.

THEOREM 2. If $H$ is the holomorph of a finitely generated abelian group $G$, and if $G$ is an invariant maximal-abelian subgroup of $H$ isomorphic to $G$, then $H$ is also the holomorph of $G$.

Proof. Clearly $H$ is the holomorph of $G$ if and only if there exists an automorphism $\eta$ of $H$ mapping $G$ onto $G$. We shall construct such an automorphism. In $\$ 9$ it was shown that if $G \neq G, G$ must be one of the groups $\bar{G}, G(z), G(q, z)$, or $G(r, s)$. We suppose first that $G$ is one of the groups $G(z)$, $G(q, z)$, or $G(r, s)$. Then we note that every element of $G$ occurs once and only once as the first component of an element of $G$. If $g \in G$, let $\left(g, \sigma_{g}\right)$ be the element of $G$ with $g$ as its first component. Now if $\tau \in A$, then

$$
(e, \tau)\left(g, \sigma_{g}\right)(e, \tau)^{-1}=\left(\tau g, \tau \sigma_{g} \tau^{-1}\right) \in G .
$$

It follows that

$$
\sigma_{\tau g}=\tau \sigma_{g} \tau^{-1} \text {. }
$$

Let $D$ be the group generated by the set of all elements of the form $\sigma_{g} h / h$, $g, h \in G$. Then it can be verified directly that if $d \in D$, then $d^{2}=e, \sigma_{0} d=d$ for all $g \in G$, and $D \subseteq G$. Put

$$
\eta f=\left(f, \sigma_{f}\right)
$$

if $f=g_{i}, f=\alpha, f=\beta, f \in F_{2}$, or if $f \in G(p), p \neq 2$. It is clear that (33) determines an isomorphism $\eta$ of $G$ onto $G$. For any $g \in G$, let $\eta g=\left(g^{*}, \tau_{g}\right)$. Then $g \rightarrow g^{*}$ is a one-to-one mapping of $G$ onto itself and $g^{*} / g \in D \subseteq G$ for all $g \in G$. Hence $\left(g / g^{*}\right)\left(g^{*}, \tau_{g}\right)=\left(g, \tau_{g}\right) \in G$. It follows that $\tau_{g}=\sigma_{g}$ and $\eta g=\left(g^{*}, \sigma_{g}\right)$. Also if 
$d \in D$ and if $g$ and $h$ are elements of $G$, then $(g d)^{*}=g^{*} d, d^{*}=d$, and $\sigma_{g} h^{*} / h^{*}$ $=\sigma_{\vartheta} h / h$. Now

$$
\eta(g h)=\eta g \eta h=\left(g^{*}, \sigma_{\vartheta}\right)\left(h^{*}, \sigma_{h}\right)=\left(g^{*} \sigma_{\vartheta} h^{*}, \sigma_{\vartheta} \sigma_{h}\right) .
$$

Therefore

$$
(g h)^{*}=g^{*} \sigma_{g} h^{*}=g^{*} h^{*}\left(\sigma_{g} h^{*} / h^{*}\right)=g^{*} h^{*}\left(\sigma_{g} h / h\right) .
$$

Hence

$$
g^{*} h^{*}=(g h)^{*}\left(\sigma_{g} h / h\right)=\left\{g h\left(\sigma_{g} h / h\right)\right\}^{*}=\left(g \sigma_{g} h\right)^{*} .
$$

Now if $\mu \in A$, let $\mu^{*}$ be the mapping defined by $\mu^{*} g^{*}=(\mu g)^{*}$. Then $\mu^{*}$ is a one-to-one mapping of $G$ onto itself. By (32) and (34)

$$
\begin{aligned}
(\mu g)^{*}(\mu h)^{*} & =\left(\mu g \sigma_{\mu g} \mu h\right)^{*}=\left(\mu g \mu \sigma_{g} h\right)^{*}=\left\{\mu\left(g \sigma_{g} h\right)\right\}^{*} \\
& =\mu^{*}\left(g \sigma_{g} h\right)^{*}=\mu^{*}\left(g^{*} h^{*}\right) .
\end{aligned}
$$

Hence $\mu^{*} \in A$. It is easily shown that $\mu^{* *}=\mu$ and that $\mu^{*} \nu^{*}=(\mu \nu)^{*}$. Hence the mapping $\mu \rightarrow \mu^{*}$ is an automorphism of $A$. Now the first component of $\left(e, \mu^{*}\right)(\eta h)\left(e, \mu^{*}\right)^{-1}$ is $\mu^{*} h^{*}=(\mu h)^{*}$. It follows that

$$
\left(e, \mu^{*}\right)(\eta h)\left(e, \mu^{*}\right)^{-1}=\eta \mu h .
$$

We now define $\eta(g, \mu)=\eta g\left(e, \mu^{*}\right)$. Clearly $\eta$ is a one-to-one mapping of $H$ onto itself, sending $G$ onto $G$. Furthermore by (35)

$$
\begin{aligned}
\eta(g, \mu) \eta(h, \nu) & =\eta g\left(e, \mu^{*}\right) \eta h\left(e, \nu^{*}\right)=\eta g \eta \mu h\left(e, \mu^{*}\right)\left(e, \nu^{*}\right) \\
& =\eta(g \mu h)\left(e,(\mu \nu)^{*}\right)=\eta\{(g, \mu)(h, \nu)\} .
\end{aligned}
$$

Hence $\eta$ is an automorphism of $H$.

There remains the case $G=\bar{G}$. Here $u=4, v=2, w=1, k=0$. Define

$$
\eta \alpha=(\beta, \psi), \quad \eta \beta=(e, \omega), \quad \text { and } \eta f=f \quad \text { if } f \in F(2) .
$$

Then $\eta$ is an isomorphism of $G$ onto $G$. We seek to extend $\eta$ to an automorphism of $H$. Now $A$ is the direct product of $A(2)$ and $A^{\prime}(2)$, where $A(2)$ consists of those automorphisms leaving every element of $F(2)$ fixed, and $A^{\prime}(2)$ consists of those automorphisms leaving every element of $G(2)$ fixed. Furthermore $A(2)$ is generated by $\psi$ and an automorphism $\zeta$ of order 4 such that $\zeta \alpha=\alpha \beta, \zeta \beta=\alpha^{2} \beta$. It can be verified directly that (36),

$$
\eta(e, \zeta)=(\alpha, \zeta), \quad \eta(e, \psi)=(\alpha \beta, \psi)
$$

and

$$
\eta(e, \tau)=(e, \tau) \quad \text { if } \quad \tau \in A^{\prime}(2)
$$

determine an automorphism $\eta$ of $H$ mapping $G$ onto $G$. This completes the proof. 
Corollary. If $H$ is the holomorph of a finite abelian group $G$, and if $G$ is an invariant subgroup of $H$ isomorphic to $G$, then $H$ is also the holomorph of $G$.

Proof. It has been shown that under these conditions $G$ is a maximal abelian subgroup of $H$. Hence Theorem 2 applies.

Theorem 2 and the corollary to Theorem 1 give us the following:

TheOREм 3. Let $G$ and $G^{\prime}$ be finitely generated abelian groups and let $H$ be the holomorph of $G$. Then $H$ is the holomorph of $G^{\prime}$ if and only if $G^{\prime}$ is an invariant maximal-abelian subgroup of $H$ isomorphic to $G$. If $G$ is a finite group then $H$ is the holomorph of $G^{\prime}$ if and only if $G^{\prime}$ is an invariant subgroup of $H$ isomorphic to $G$.

When either of the groups $G$ and $G^{\prime}$ is not abelian the problem appears to be much more difficult. It is not true that two finite nonabelian groups with the same holomorph are isomorphic. For example if $n \geqq 3$, the dihedral and dicyclic groups of order $4 n$ have the same holomorph.

\section{REFERENCES}

1. G. A. Miller, On the multiple holomorphs of a group, Math. Ann. vol. 66 (1908) pp. 133142.

2. Yu. A. Gol'fand, On the group of automorphisms of the holomorph of a group, Rec. Math. (Mat. Sbornik) N.S. vol. 27 (1950) pp. 333-350.

YALE UNIVERSITY,

New Haven, Conn. 